\title{
The Autoregressive Conditional Marked Duration Model: Statistical Inference to Market Microstructure
}

\author{
Simon Sai Man Kwok, Wai Keung Li and Philip Leung Ho Yu \\ The University of Hong Kong
}

\begin{abstract}
We consider the Autoregressive Conditional Marked Duration (ACMD) model and apply it to 16 stocks traded in Hong Kong Stock Exchange (SEHK). By examining the orderings of appropriate sets of model parameters, market microstructure phenomena can be explained. To substantiate these conclusions, likelihood ratio test is used for testing the significance of the parameter orderings of the ACMD model. While some of our results resolve a few controversial market microstructure hypotheses and echo some of the existing empirical evidence, we discover some interesting market microstructure phenomena that may be characteristic to SEHK.
\end{abstract}

Key words: Autoregressive conditional duration, likelihood ratio test, market microstructure, statistical inference.

\section{Introduction}

Easley and O'Hara (1992) are among the first to propose the idea that trade duration, or the length of the time interval between successive transactions in stock market, contains valuable information. By applying a simple Bayesian model to stock market data, they demonstrated in particular that trade duration has an effect on the transaction prices of the traded stock. This motivates econometricians to search for models that can explain the next stock price movement in terms of previous trade durations. Engle and Russell (1998) proposed the Autoregressive Conditional Duration (ACD) model so that the duration clustering phenomenon can be captured and hence more accurate instantaneous volatility of stock price can be derived, but the linear assumption and the excess dispersion problem of the fitted model illustrated in the paper necessitate more complicated formulation of the ACD model which approximates the reality better. This task was carried out in two main directions: either directly generalizing the ACD model through transformation of the duration equation, or assuming that the conditional duration process is governed by different duration equations 
over time with an underlying stochastic process that switches between different regimes (see Hautsch, 2004 for a brief overview).

One line of extension of the ACD model was explored by Tay et al. (2004), who proposed the Autoregressive Conditional Marked Duration (ACMD) model. This model allows us to analyze marked duration processes. Some examples of marks are price movement indicator and trade initiation indicator. In contrast to the original ACD model, the ACMD model assumes that the occurrence of transaction events is the result of competition among multiple underlying stochastic marked duration processes which are independent of one another. Each of the underlying duration processes is associated with one of the states of a prescribed mark, and its dynamics resembles that of an ACD model.

One of the major applications of the ACMD model arises from its flexibility: it can be augmented by other market variables and interaction terms. As a result, market microstructure phenomena can be easily explained within the ACMD framework because we can tell how the mark associated with the next transaction is related to the observed market variables, the realized durations and also their interactions. The relevant conclusions can be revealed from the estimated parameters of the model. In particular, some market microstructure hypotheses, such as whether low trading intensity is associated with bad news or simply the absence of private information, can be readily examined within the ACMD framework. We apply this model to 16 stocks traded in the stock market of Hong Kong. Some empirical phenomena local to the Hong Kong stock market are uncovered. In particular, it is interesting to find that investing in some stocks may be less susceptible to short-sale constraints than investing in other stocks and hence the investors of those stocks may capitalize on their knowledge of bad news as efficiently as their knowledge of good news. This tends to conform to Easley and O'Hara's (1992) idea that low trading intensity simply indicates the absence of private information. In contrast, the results of Tay et al. (2004) provided a unanimous support to the alternative hypothesis that low trading intensity means bad news (Diamond and Verrecchia, 1987).

We start by formulating the ACMD model in section 2. We explain how to obtain and process the data to be fitted by the ACMD model in section 3 . The empirical results are reported in section 4 , and the conclusion is in section 5 .

\section{The ACMD Model}

\subsection{Model Formulation}

Our data set contains the time of occurrence of $N+1$ events (transactions). Denote the time of occurrence of the $i$ th event by $t_{i}, i=0,1, \ldots, N$. First we choose the mark associated with $\left\{t_{i}\right\}$. The mark has $m$ discrete states. The 
state of the mark observed when the $i$ th event occurs is denoted by $W_{i}$ (and its realization by $\left.w_{i}\right), i=1,2, \ldots, N$. In our application the mark is a price movement indicator with $m=3$, indicating there is a price increase, a price decrease or no price change at the current transaction relative to the previous one. Other exogenous variables can be augmented to the model, depending on the hypotheses we want to examine. In our financial application, we include the volume of transaction and the trade initiation indicator (see section 2.2). The vector of exogenous variables observed when the $i$ th event occurs is denoted by $\boldsymbol{v}_{i}, i=1,2, \ldots, N$.

Denote the information set after the $i$ th event by $\Phi_{i}=\left\{t_{r}, w_{r}, \boldsymbol{v}_{r}, r=\right.$ $1,2, \ldots, i\}$. Let the time duration between the $i$ th and $(i-1)$ th events be $X_{i}, i=1,2, \ldots, N$. Its realization $x_{i}$ can be obtained from the sequence of event occurrence in the data set. (To be precise, $\tilde{\tilde{x}}_{i}=t_{i}-t_{i-1}$, and $\left\{x_{i}\right\}$ is obtained from $\left\{\tilde{\tilde{x}}_{i}\right\}$ after data cleansing and diurnal adjustment; see sections 3.2 and 3.3.) The role of the mark chosen above is to classify each event occurrence so that we can form $m$ underlying stochastic duration processes, each of which is associated with one of the $m$ states of the mark.

Let $T_{j i}$ be the random time duration (in seconds) between the $(i-1)$ th and the $i$ th events when state $j$ of the mark is observed. Conditional on the past information set $\Phi_{i-1}$ after the $(i-1)$ th event, all these $m$ marked durations are assumed to be independent over all states of the mark. That is, $T_{j i} \mid \Phi_{i-1}$ are independent over all $j$. The corresponding density function $f_{T_{j i} \mid \Phi_{i-1}}\left(x_{i}\right)$, distribution function $F_{T_{j i} \mid \Phi_{i-1}}\left(x_{i}\right)$ and survival function $S_{T_{j i} \mid \Phi_{i-1}}\left(x_{i}\right)$ are assumed to depend on the conditional intensity function $\lambda_{j i}$. In particular, if the conditional marked duration $T_{j i} \mid \Phi_{i-1}$ follows an exponential distribution, then $\lambda_{j i}$ is the reciprocal of the conditional expected marked duration $\psi_{j i}$. Given $\Phi_{i-1}$, each of the conditional expected marked duration $\psi_{j i}$ is updated according to the dynamics resembling that of an ACD model (see section 2.2).

After choosing the appropriate distribution for the underlying conditional marked duration $T_{j i} \mid \Phi_{i-1}$, we can then derive the conditional joint distribution of the mark and the unmarked duration, conditional on the past information. The conditional independence assumption on the marked durations plays a crucial role in linking the latter to the former and thus enables us to construct the loglikelihood function. Conditional on $\Phi_{i-1}$, the joint distribution of the mark, $W_{i}$, and the unmarked duration to the $i$ th transaction, $X_{i}$, is given by:

$$
\begin{aligned}
p_{i}\left(k, x_{i} \mid \Phi_{i-1}\right) & =\operatorname{Pr}\left(W_{i}=k \text { and } X_{i}=x_{i} \mid \Phi_{i-1}\right) \\
& =\operatorname{Pr}\left(W_{i}=k \text { and } T_{W_{i} i}=x_{i} \mid \Phi_{i-1}\right)
\end{aligned}
$$




$$
\begin{aligned}
& =\operatorname{Pr}\left(W_{i}=k \text { and } T_{k i}=x_{i} \mid \Phi_{i-1}\right) \\
& =\operatorname{Pr}\left(\left[\bigcap_{j \neq k}\left\{T_{j i}>x_{i}\right\}\right] \bigcap\left\{T_{k i}=x_{i}\right\} \mid \Phi_{i-1}\right) \\
& =\prod_{j \neq k} S_{T_{j i} \mid \Phi_{i-1}}\left(x_{i}\right) \cdot f_{T_{k i} \mid \Phi_{i-1}}\left(x_{i}\right) \\
& =\prod_{j \in \Omega} S_{T_{j i} \mid \Phi_{i-1}}\left(x_{i}\right) \cdot \frac{f_{T_{k i} \mid \Phi_{i-1}}\left(x_{i}\right)}{S_{T_{k i} \mid \Phi_{i-1}}\left(x_{i}\right)}
\end{aligned}
$$

for all $k \in \Omega=\left\{k_{1}, \ldots, k_{m}\right\}$. Note that $(2.1)$ holds because $\left\{W_{i}=k\right\}=\left\{T_{k i}<\right.$ $T_{j i}$, for $\left.j \neq k\right\}$, and (2.2) follows because of the independence assumption on $T_{j i} \mid \Phi_{i-1}$ over $j$. Note also that $p_{i}\left(k, x_{i} \mid \Phi_{i-1}\right)=\operatorname{Pr}\left(W_{i}=k\right.$ and $\left.T_{k i}=x_{i} \mid \Phi_{i-1}\right)$ is not equal to $\operatorname{Pr}\left(T_{k i}=x_{i} \mid \Phi_{i-1}\right)$, as $\left\{T_{k i}=x_{i} \mid \Phi_{i-1}\right\} \not \subset\left\{T_{j i}>x_{i}\right.$, for $\left.j \neq k \mid \Phi_{i-1}\right\}$ in general, unless the condition $W_{i}=k$ is true (see (2.1)).

The log-likelihood function for the ACMD model for an observed sequence of $\left\{w_{i}, x_{i}\right\}, i=1, \ldots, N$ is given by

$$
L L_{N}=\sum_{i=1}^{N} \ln p_{i}\left(w_{i}, x_{i} \mid \Phi_{i-1}\right)=\sum_{i=1}^{N}\left[\sum_{j \in \Omega} \ln S_{T_{j i} \mid \Phi_{i-1}}\left(x_{i}\right)+\ln \frac{f_{T_{w_{i}, i} \mid \Phi_{i-1}}\left(x_{i}\right)}{S_{T_{w_{i}, i} \mid \Phi_{i-1}}\left(x_{i}\right)}\right]
$$

If $T_{j i} \mid \Phi_{i-1}$ follows an exponential distribution with parameter $\lambda_{j i}$, then

$$
p_{i}\left(k, x_{i} \mid \Phi_{i-1}\right)=\lambda_{k i} \exp \left\{-\left(\sum_{j \in \Omega} \lambda_{j i}\right) x_{i}\right\} .
$$

We can obtain the explicit form of the conditional marginal density of the unmarked duration to the $i$ th event, $X_{i}$ :

$$
f_{X_{i}}\left(x \mid \Phi_{i-1}\right)=\sum_{j \in \Omega} p_{i}\left(j, x \mid \Phi_{i-1}\right)=\left(\sum_{j \in \Omega} \lambda_{j i}\right) \exp \left\{-\left(\sum_{j \in \Omega} \lambda_{j i}\right) x\right\},
$$

and that of the conditional marginal probability function of the winning state at the $i$ th event, $W_{i}$ :

$$
f_{W_{i}}\left(k \mid \Phi_{i-1}\right)=\int_{0}^{\infty} p_{i}\left(k, x \mid \Phi_{i-1}\right) d x=\frac{\lambda_{k i}}{\sum_{j \in \Omega} \lambda_{j i}} .
$$

In fact, we can interpret the realized conditional unmarked duration as the shortest of the $m$ conditional marked durations. Indeed, by comparing (2.4) 
with the distribution of $T_{j i} \mid \Phi_{i-1}$, it can be deduced that, given $\Phi_{i-1}, X_{i}=$ $\min _{j \in\left\{k_{1}, \ldots, k_{m}\right\}}\left\{T_{j i}\right\}$. Note that under the exponential distribution, $X_{i} \mid \Phi_{i-1}$ and $W_{i} \mid \Phi_{i-1}$ are independent, as $p_{i}\left(k, x \mid \Phi_{i-1}\right)=f_{X_{i}}\left(x \mid \Phi_{i-1}\right) \cdot f_{W_{i}}\left(k \mid \Phi_{i-1}\right)$. This also holds if we replace the exponential distribution by the Weibull distribution with parameters $\lambda_{j i}$ and $\phi$. In this case the joint distribution is given by

$$
p_{i}\left(k, x_{i} \mid \Phi_{i-1}\right)=\phi x_{i}^{\phi-1} \lambda_{k i}^{\phi} \exp \left\{-\left(\sum_{j \in \Omega} \lambda_{j i}^{\phi}\right) x_{i}^{\phi}\right\} .
$$

The conditional marginal distributions of $X_{i}$ and $W_{i}$ are then given by

$$
f_{X_{i}}\left(x \mid \Phi_{i-1}\right)=\sum_{j \in \Omega} p_{i}\left(j, x \mid \Phi_{i-1}\right)=\phi x^{\phi-1}\left(\sum_{j \in \Omega} \lambda_{j i}^{\phi}\right) \exp \left\{-\left(\sum_{j \in \Omega} \lambda_{j i}^{\phi}\right) x^{\phi}\right\}
$$

and

$$
f_{W_{i}}\left(k \mid \Phi_{i-1}\right)=\int_{0}^{\infty} p_{i}\left(k, x \mid \Phi_{i-1}\right) d x=\frac{\lambda_{k i}^{\phi}}{\sum_{j \in \Omega} \lambda_{j i}^{\phi}}
$$

\subsection{The dynamics}

The conditional expected marked durations $\psi_{j i}$ are governed by $m$ individual ACD models. We denote the state $k$ indicator for the mark by $D_{k}(z)$ which takes on value 1 if $z=k$ and 0 otherwise, $k \in \Omega$. Specifically, $\psi_{j i}$ follows the dynamics:

$$
\ln \psi_{j i}=\sum_{k \in \Omega} v_{j k} D_{k}\left(w_{i-1}\right)+\alpha_{j} \ln \psi_{j, i-1}+\beta_{j} \ln x_{i-1}+f\left(x_{i-1}, \boldsymbol{v}_{i-1} ; \boldsymbol{\rho}_{j}\right)
$$

for all $j \in \Omega$. The function $f$ is linear in the (natural log of the) exogenous variables in $\boldsymbol{v}$ and possibly their product with $x_{i-1}$, each term weighed by the parameters in $\boldsymbol{\rho}_{j}$.

In our application, we use a three-state price movement indicator as the mark of the ACMD model. The three marks are downtick, no price change and uptick and are represented by the state indices $k_{1}=-1, k_{2}=0, k_{3}=1$ respectively. We include the transaction volume in lots, $s_{i}$, and the trade initiation indicator, $y_{i}$, as our set of exogenous variables $\boldsymbol{v}_{i}$. The trade initiation indicator $y_{i}$ takes on the value 1 if the $i$ th transaction is a buyer-initiated trade and 0 if it is a seller-initiated trade. After the $i$ th transaction, the conditional expected marked trade duration will be updated as follows:

$$
\ln \psi_{j i}=\sum_{k=-1}^{1} v_{j k} D_{k}\left(w_{i-1}\right)+\alpha_{j} \ln \psi_{j, i-1}+\beta_{j} \ln x_{i-1}+\gamma_{j} \ln s_{i-1}+\varphi_{j} y_{i-1}
$$




$$
+\theta_{j}\left(y_{i-1} \ln s_{i-1}\right)+\eta_{j}\left(y_{i-1} \ln x_{i-1}\right)+\xi_{j}\left(y_{i-1} \ln x_{i-1} \ln s_{i-1}\right)
$$

for all $j \in \Omega$. The last three interaction terms are included to facilitate the study of the joint effects of the past exogenous variables $\boldsymbol{v}_{i-1}$ and realized diurnally adjusted durations $x_{i-1}$ (see section 3.3 for details on diurnal adjustment) on the likelihood of the next realized states of the mark. The marginal effect on the conditional expected duration $\psi_{j i}$ is measured by the corresponding coefficient associated to that exogenous variable.

Inference regarding various market microstructure hypotheses, which predict how the price probably moves in a given scenario conditional on the realizations of market variables, trade durations and their interactions, can be made by examining the relative magnitude of appropriate sets of estimated coefficients (see section 4).

\section{Data}

\subsection{Data description}

The ACMD model is applied to the intraday transaction data of some Hong Kong stocks. Sixteen stocks with leading market capitalization in the four sectors (property, utility, commerce and finance) were selected (see Table 1). The sample period for all stocks encompasses 121 trading days from 1 January 2003 to 30 June 2003. There are two separate trading periods in each normal trading day in the Hong Kong Stock Exchange: the morning session from 10:00 to 12:30 and the afternoon session from 14:30 to 16:00.

Table 1: Facts about the 16 stocks in the sample

\begin{tabular}{cllc}
\hline & & & $\begin{array}{c}\text { Market capitalization } \\
\text { as at Dec 2004 } \\
\text { (in billion HKD) }\end{array}$ \\
\hline Stock & \multicolumn{1}{c}{ Stock name } & Industry & 167 \\
2 & Cheung Kong (Holdings) Ltd. & property & 106 \\
3 & CLP Holdings Ltd. & utility & 90 \\
4 & The Wong and China Gas Company Ltd. & utility & 62 \\
5 & HSBC Holdings plc & commerce & 1388 \\
6 & Hongkong Electric Holdings Ltd. & finance & 74 \\
8 & Pacific Century CyberWorks Ltd. & utility & 26 \\
11 & Hang Seng Bank Ltd. & commerce & 201 \\
12 & Henderson Land Development Company Ltd. & property & 67 \\
13 & Hutchison Whampoa Ltd. & commerce & 295 \\
16 & Sun Hung Kai Properties Ltd. & property & 173 \\
17 & New World Development Company Ltd. & property & 26 \\
19 & Swire Pacific Ltd. (A share) & commerce & 57 \\
23 & The Bank of East Asia & finance & 34 \\
267 & CITIC Pacific Ltd. & commerce & 45 \\
941 & China Mobile (Hong Kong) Ltd. & commerce & 479 \\
\hline
\end{tabular}




\subsection{Data extraction and cleansing}

From the Trade Record released by the Hong Kong Exchanges and Clearing Limited, the sequences of trade duration (in seconds), $\left\{\tilde{\tilde{x}}_{i}\right\}$, the price change indicator, $\left\{\tilde{w}_{i}\right\}$, and the volume traded (in lots), $\left\{\tilde{s}_{i}\right\}$, can be directly obtained (the tilde above the variable indicates that the sequence is raw and has not undergone data cleansing). The sequence of trade initiation indicator, $\left\{\tilde{y}_{i}\right\}$, is derived from both the Trade Record and the Bid and Ask Record using the simplest version of the Lee and Ready (1991) algorithm.

Those transactions that occur before the opening (10:00) and after the close (16:00) of the stock exchange are dropped from the analysis. To mitigate the opening auction effect, we also ignore the transactions that occurred in the first 20 minutes of each trading day (10:00 to 10:20). Then we take the average of the trade durations associated with transactions in the following 10 minutes (10:20 to 10:30) and use it as the starting trade duration associated with the first trade after 10:30 (Engle and Russell, 1998). The total number of transactions, after data filtering, ranges from 20101 (stock 23: The Bank of East Asia) to 111590 (stock 5: HSBC Holdings plc). After data cleansing, we obtained four filtered data series: the filtered trade duration series $\left\{\tilde{x}_{i}\right\}$, the price movement indicator series $\left\{w_{i}\right\}$, the volume series $\left\{s_{i}\right\}$ and the trade initiation series $\left\{y_{i}\right\}$.

\subsection{Diurnal adjustment}

Trade duration is one of the proxies of trade activity as it measures how often trade arrivals occur. On the other hand, trade activity has a fixed pattern of highs and lows for any generic trading day: we often observe concentrated trade activity at the start of a trading session and towards the end of a trading session, and little trade activity near the middle of a trading session. In order to have a more meaningful analysis as to how the variation of trade activity of a particular day compares to that of a generic trading day, it is suggested that trade duration be decomposed into a deterministic part as well as a stochastic part (Engle and Russell, 1998), so that the stochastic part would be a proxy for the relative trade activity.

The deterministic part is approximately captured by the diurnal factor function $\tilde{\phi}(t)$ which can be obtained by fitting two cubic splines to the filtered trade duration series for the morning session and the afternoon session. The International Mathematical and Statistical Library (IMSL) subroutine CSPLINE is employed to obtain the two cubic splines. The resulting diurnally adjusted trade duration from the $(i-1)$ th transaction (which occurred at $t_{i-1}$ ) to the $i$ th transaction (which occurred at $t_{i}$ ), $x_{i}$, is thus obtained by dividing the filtered trade duration from the $(i-1)$ th transaction to the $i$ th transaction, $\tilde{x}_{i}$, by the scaled 
diurnal factor associated with the time point at which the $(i-1)$ th transaction occurs. That is, $x_{i}=\tilde{x}_{i} / \phi\left(t_{i-1}\right)$, where $\phi(t)=c \tilde{\phi}(t)$ is the diurnal factor function scaled by a constant $c$ such that the sample mean of the diurnally adjusted trade durations is equal to the sample mean of the filtered trade durations: $\bar{x}_{i} \triangleq \overline{\tilde{x}}_{i}$. In this manner, the unit of the trade duration series is preserved after diurnal adjustment. Figure 1 shows the graphs of $\phi_{t}$ for the morning and afternoon sessions for Cheung Kong (Holdings) Ltd. (stock 1).

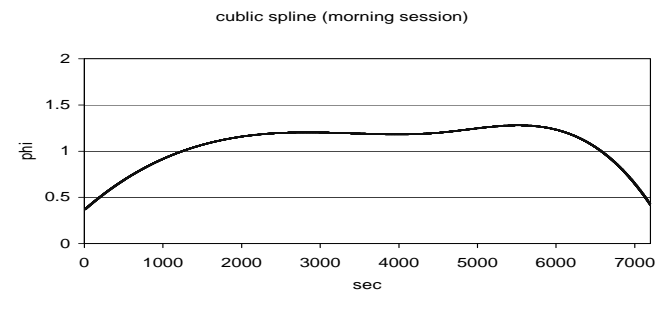

(a)

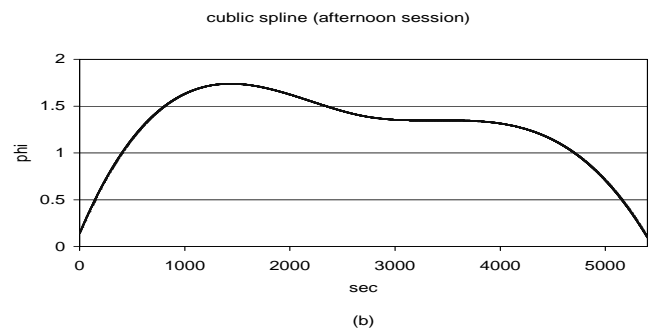

Figure 1: The cublic spline of stock 1 in the morning session (a) and in the afternoon session (b)

\section{Discussion}

Now, we will discuss the implications to market microstructure phenomena one by one as revealed by the parameter orderings of the ACMD models fitted to the stock price data of the 16 stocks in our sample. Likelihood ratio tests are carried out to substantiate our conclusions. To simplify the analysis, we will make inference on whether an uptick or a downtick will have a higher probability to be observed in a given scenario, conditional on the past information set and that the price actually changes. By formulating hypotheses in this manner, we can concentrate on the relative magnitude of a pair of coefficients, one associated with the uptick state and the other associated with the downtick state, instead of having to examine the ordering, and the significance of the ordering, of three 
coefficients, each of which associated with one of the three states of the mark. As an illustration, if the estimated $\beta_{-1}$ happens to be smaller than the estimated $\beta_{1}$, then, given a long realized trade duration $x_{i-1}$ since the last transaction, the conditional expected duration associated with the downtick state, $\psi_{-1, i}$, decreases relative to that associated with the uptick state, $\psi_{1, i}$. Equivalently, the trade intensity associated with downtick, $\lambda_{-1, i}$, increases relative to that associated with uptick, $\lambda_{1, i}$, meaning that there is an increased probability to observe a downtick than an uptick at the next transaction following a trade that happened a long time ago. Therefore, we will apply one linear constraint for each restricted model and hence the likelihood ratio test statistics should follow a chi-square distribution approximately with one degree of freedom.

To save space, we only display the parameter estimates of the ACMD model for stock 5 (see Table 2, detailed estimates for all stocks are available upon request). The results of the likelihood ratio tests for all stocks are summarized in Table 3. All the tests were conducted at the 5\% significance level.

Table 2: Parameter estimates of ACMD model for HSBC Holdings plc (stock 5)

\begin{tabular}{cllccc}
\hline & $j=-1$ & $j=0$ & $j=1$ & LR & Implication \\
\hline$\nu_{j,-1}$ & 3.199 & 0.596 & 0.970 & 274.946 & up \\
& $(0.0182)$ & $(0.0051)$ & $(0.0109)$ & & down \\
$\nu_{j, 0}$ & 1.340 & 0.148 & 3.267 & 371.978 & down \\
& $(0.0043)$ & $(0.0008)$ & $(0.0097)$ & & \\
$\nu_{j, 1}$ & -0.832 & 0.199 & 4.661 & 2705.996 & N /A \\
& $(0.0094)$ & $(0.0049)$ & $(0.0427)$ & & \\
$\alpha_{j}$ & 0.678 & 0.816 & 0.343 & 311.060 & down \\
& $(0.0006)$ & $(0.0003)$ & $(0.0010)$ & & \\
$\beta_{j}$ & 0.195 & 0.179 & 0.372 & 192.150 & down \\
& $(0.0013)$ & $(0.0004)$ & $(0.0025)$ & & \\
$\gamma_{j}$ & -0.016 & 0.012 & 0.030 & 11.180 & down \\
& $(0.0029)$ & $(0.0007)$ & $(0.0049)$ & & up \\
$\psi_{j}$ & -0.022 & -0.008 & 0.564 & 318.014 & down \\
$\theta_{j}$ & $(0.0045)$ & $(0.0008)$ & $(0.0075)$ & & \\
& 0.019 & 0.004 & -0.038 & 10.064 & down \\
$\eta_{j}$ & $(0.0036)$ & $(0.0009)$ & $(0.0053)$ & & \\
$\xi_{j}$ & -0.060 & 0.001 & 0.122 & 187.596 & \\
& $(0.0021)$ & $(0.0005)$ & $(0.0031)$ & & \\
& 0.000 & 0.002 & 0.026 & 20.426 & \\
\hline
\end{tabular}

$\dagger$ All LR tests significant at the $5 \%$ significance level. Standard error of the estimates in brackets. 
Table 3: Summary of stocks exhibiting significant price movement under various cases based on likelihood ratio test

\begin{tabular}{cll}
\hline \multicolumn{1}{c}{ Cases } & Implication & Stocks \\
\hline$\nu_{-1,-1}>\nu_{1,-1}$ & downtick $\rightarrow$ uptick & all \\
$\nu_{-1,-1}<\nu_{1},-1$ & downtick $\rightarrow$ downtick & none \\
$\nu_{-1,1}>\nu_{1,1}$ & uptick $\rightarrow$ uptick & none \\
$\nu_{-1,1}<\nu_{1,1}$ & uptick $\rightarrow$ downtick & all \\
$\beta_{-1}>\beta_{1}$ & long duration $\rightarrow$ uptick & none \\
$\beta_{-1}<\beta_{1}$ & long duration $\rightarrow$ downtick & $2,3,5,6,8,11,13,17,23$ \\
$\gamma_{-1}>\gamma_{1}$ & large trade $\rightarrow$ uptick & 11 \\
$\gamma_{-1}<\gamma_{1}$ & large trade $\rightarrow$ downtick & $2,3,4,5,6,13$ \\
$\varphi_{-1}>\varphi_{1}$ & buy trade $\rightarrow$ uptick & 4 \\
$\varphi_{-1}<\varphi_{1}$ & buy trade $\rightarrow$ downtick & $2,3,5,6,8,11,13,16,17$, \\
$\theta_{-1}>\theta_{1}$ & large buy trade $\rightarrow$ uptick & 267,941 \\
$\theta_{-1}<\theta_{1}$ & large buy trade $\rightarrow$ downtick & $1,2,3,5,8,11,13,16,17$, \\
$\eta_{-1}>\eta_{1}$ & buy trade after long time $\rightarrow$ uptick & 267 \\
$\eta_{-1}<\eta_{1}$ & buy trade after long time $\rightarrow$ downtick & 4 \\
$\xi_{-1}>\xi_{1}$ & large buy trade after long time $\rightarrow$ uptick & none \\
$\xi_{-1}<\xi_{1}$ & large buy trade after long time $\rightarrow$ downtick & none \\
\hline
\end{tabular}

The first observation is that $\nu_{-1,-1}>\nu_{1,-1}$ and $\nu_{1,1}>\nu_{-1,1}$ significantly and uniformly for all of the stocks being considered. In other words, a downtick (uptick) entails a higher probability of an uptick (downtick). This is no coincidence because all stock price series exhibit the common bid-ask bounce phenomenon.

As for the coefficients of duration, the results are mixed. At first sight, all except stocks 267 and 941 share the same ordering of beta: $\beta_{1}>\beta_{-1}$. A more careful judgment supplemented by the likelihood ratio test with the null $\beta_{1}=\beta_{-1}$ reveals that we cannot reject the null that $\beta_{1}=\beta_{-1}$ for stocks $1,4,12,16,19$, 267 and 941, while we can reject the null for the rest. It is interesting to note that stocks 1, 12 and 16 are issued by leading property developers in Hong Kong. In contrast to the findings of Tay et al. (2004), it seems that these seven stocks do not support the view by Diamond and Verrecchia (1987) that a delay in trading (a drop in stock price) entails the existence of bad news due to short-sale constraints. In other words, it is well possible that the investors of some stocks (such as the seven stocks with insignificant difference of $\beta_{-1}$ and $\beta_{1}$ ) seem to be unaffected by short-sale constraints and can capitalize on their knowledge of bad news as efficiently as their knowledge of good news. The results of these stocks tend to support more of Easley and O'Hara's (1992) idea that no news simply means no private information.

The ordering of $\gamma$ 's varies from stock to stock: $\gamma_{-1}>\gamma_{1}$ is observed for stocks 1,11 and 12 while $\gamma_{1}>\gamma_{-1}$ is observed for the rest. After conducting likelihood ratio tests, however, we find that the difference between $\gamma_{-1}$ and $\gamma_{1}$ are significant only for stocks $2,3,4,5,6,11$ and 13 . The orderings are not significant for the 
other stocks. Tay et al. (2004) also obtained an ambiguous result in this respect. This indicates that, overall speaking, trade volume alone does not consistently infer a particular direction of the next price movement.

We discover that $\varphi_{-1}>\varphi_{1}$ for stocks 4, 12 and 19, compared to $\varphi_{1}>\varphi_{-1}$ for the remaining stocks. All of their likelihood ratio tests, except for stocks 1 , 12,19 and 23, reject the null hypotheses that $\varphi_{1}=\varphi_{-1}$. Among the 12 stocks whose difference between $\varphi_{-1}$ and $\varphi_{1}$ is significant, only stock 4 has $\varphi_{-1}>\varphi_{1}$. This may suggest that, holding all other factors constant, a buy trade induces a higher probability of a subsequent downtick. This is consistent with Tay et al. (2004) who obtained the same findings for all of their five stocks in the sample.

All stocks exhibit the ordering $\theta_{-1}>\theta_{1}$ except for stocks 4 and 941. All of these orderings, except for stocks $6,12,19,23$ and 941 , are statistically significant as revealed by likelihood ratio tests. Among the 11 stocks whose difference between $\theta_{-1}$ and $\theta_{1}$ is significant, only stock 4 has $\theta_{1}>\theta_{-1}$. This implies that given the past information and that the price will really change at the next transaction, a large buy trade scenario is most probably followed by an uptick, which is similar to Tay et al. (2004) who reported a uniform ordering $\theta_{-1}>\theta_{1}$ for all of their five stocks.

We also observe that $\eta_{1}>\eta_{-1}$ uniformly for all stocks, same as that noted by Tay et al. (2004). Likelihood ratio tests with the null of $\eta_{1}=\eta_{-1}$ strongly confirm the significance of all the orderings. Therefore, a buy trade after a long duration induces a higher probability of downtick, echoing what Tay et al. (2004) and Dufour and Engle (2000) found.

Lastly, all stocks share the same ordering $\xi_{1}>\xi_{-1}$ except for stocks 4 and 13. The orderings are statistically significant for stocks $1,2,3,5,6,11$ and 267 only, but they share the same ordering $\xi_{1}>\xi_{-1}$ unanimously. In contrast to the findings of Tay et al. (2004), it is surprising that a large buy trade after a long duration does not induce a significantly higher probability of a subsequent uptick for all of our stocks.

\section{Conclusion}

We applied the ACMD model that uses a three-state price movement indicator as the mark to the transaction data of 16 stocks traded in the Hong Kong Stock Exchange over a half-year trading period. The results shed light on some of the market microstructure phenomena that are characteristic to the stock market of Hong Kong. We have also implemented likelihood ratio tests on the equality of chosen pairs of parameters within the ACMD framework, so that we are able to exercise more careful judgment on market microstructure hypotheses by justifying each of the comparisons made based on an appropriate likelihood ratio test. Some empirical results we obtained are different from those of Tay et al. (2004). This 
may imply that some market microstructure phenomena exhibited by Hong Kong stocks are different from those exhibited by stocks traded in NYSE. Indeed, while Tay et al. (2004) reported that large buy trades after a long duration entail a higher chance of observing a price increase for all of their five stocks, our empirical results reveal that it is not necessarily so. It is even more interesting to note from our results that investors may benefit from their knowledge of bad news as quickly as from their knowledge of good news by investing in certain stocks, such as those issued by leading property developers. This may be a manifestation of the idea, originated by Easley and O'Hara (1992), that low trading intensity indicates the absence of private information. As some aspects of our empirical results regarding market microstructure vary from stock to stock, it may be worthwhile for future research to conduct more comprehensive studies on how market microstructure phenomena differ among stocks from different industries.

\section{References}

Diamond, D. W. and R. E. Verrecchia, 1987, Constraints on short-selling and asset price adjustment to private information, Journal of Financial Economics, 18, 227-311.

Dufour, A. and R. F. Engle, 2000, The ACD model: predictability of the time between consecutive trades, Discussion paper, ISMA Centre, University of Reading.

Easley, D. and M. O'Hara, 1992, Time and the process of security price adjustment, Journal of Finance, 47, 577-605.

Engle, R. F. and A. Lunde, 2003, Trades and quotes: a bivariate point process, Journal of Financial Econometrics, 1, 159-188.

Engle, R. F. and J. R. Russell, 1998, Autoregressive conditional duration: A new model for irregularly spaced transaction data, Econometrica, 66, 1127-1162.

Godfrey, L. G., 1988, Misspecification tests in Econometrics: the Lagrange multiplier principle and other approaches, Cambridge: Cambridge University Press.

Hautsch, N, 2004, Modelling irregularly spaced financial data: theory and practice of dynamic duration models, Berlin: Springer-Verlag.

Lee, C. M. C. and M. J. Ready, 1991, Inferring trade direction from intraday data, Journal of Finance, 46, 2, 733-746.

Russell, J. R. and R. F. Engle, 2005, A discrete-state continuous-time model of financial transactions prices and times: the ACM-ACD model. Journal of Business and Economic Statistics, 23, 2, 166-180.

Tay, A., C. Ting, Y. K. Tse and M. Warachka, 2004, Transaction-data analysis of marked durations and their implications for market microstructure. Working Paper, Singapore Management University. 
Received May 17, 2007; accepted October 9, 2007.

Simon Sai Man Kwok

Department of Statistics and Actuarial Science

The University of Hong Kong

sk528@cornell.edu

Wai Keung Li

Department of Statistics and Actuarial Science

The University of Hong Kong

hrntlwk@hkucc.hku.hk

Philip Leung Ho Yu

Department of Statistics and Actuarial Science

The University of Hong Kong

plhyu@hku.hk 